\title{
Simulation Study of Hatch Spacing and Layer Thickness Effects on Microstructure in Laser Powder Bed Fusion Additive Manufacturing using a Texture-Aware Solidification Potts Model
}

\author{
Joseph Pauza and Anthony Rollett
}

Submitted: 22 January 2021 / Revised: 4 May 2021 / Accepted: 3 August 2021 / Published online: 19 August 2021

\begin{abstract}
Microstructure control in the laser powder bed fusion additive manufacturing processes is a topic of major interest because of the submillimeter length scale at which the manufacturing process occurs. The ability to control the process at the melt pool scale allows for microstructure control that few other manufacturing techniques can match. The majority of work on microstructure control has focused on altering laser parameters to control solidification conditions (Ref (R.R. Dehoff, M.M. Kirka, W.J. Sames, H. Bilheux, A.S. Tremsin, L.E. Lowe, and S.S. Babu, Site Specific Control of Crystallographic Grain Orientation through Electron Beam Additive Manufacturing, Mater. Sci. Technol., 2014, 31(8), p 931-938. R. Shi, S.A. Khairallah, T.T. Roehling, T.W. Heo, J.T. McKeown, and M.J. Matthews, Microstructural Control in Metal Laser Powder Bed Fusion Additive Manufacturing Using Laser Beam Shaping Strategy, Acta Mater., 2020, 184, p 284-305, https://doi.org/10.1016/j.actamat.2019.11.053.)). Other machine parameters, besides the laser parameters, have also been shown to affect the microstructure of AM parts (Ref (N. Nadammal, S. Cabeza, T. Mishurova, T. Thiede, A. Kromm, C. Seyfert, L. Farahbod, C. Haberland, J.A. Schneider, P.D. Portella, and G. Bruno, Effect of Hatch Length on the Development of Microstructure, Texture and Residual Stresses in Selective Laser Melted Superalloy Inconel 718, Mater. Des., 2017, 134, p 139-150, h ttps://doi.org/10.1016/j.matdes.2017.08.049. F. Geiger, K. Kunze, and T. Etter, Tailoring the Texture of IN738LC Processed by Selective Laser Melting (SLM) by Specific Scanning Strategies, Mater. Sci. Eng. A, 2016, 661, p 240-246, https://doi.org/10.1016/j.msea.2016.03.036.)). We propose an investigation of the effects of hatch spacing and layer thickness on microstructure development in laser powder bed fusion additive manufacturing. A Monte Carlo Potts model with textured solidification capabilities is used to study the effects of these parameters on a unidirectional scan strategy. The simulation results reveal substantial changes in grain morphology as well as texture. Additionally, EVP-FFT crystal plasticity simulations were performed to evaluate the effect of the microstructural shifts on mechanical response. The conclusions from this work elucidate the effects of these parameters on part microstructure as predicted by the texture-aware solidification Potts model and inform understanding of how bulk texture is predicted by the simulation approach.
\end{abstract}

Keywords additive manufacturing, computational materials design, microstructure control, modeling and simulation

\section{Introduction}

Laser powder bed fusion (LPBF) additive manufacturing (AM) is a manufacturing technique that offers a variety of advantages over other, more traditional, manufacturing tech-

This invited article is part of a special topical focus in the Journal of Materials Engineering and Performance on Additive Manufacturing. The issue was organized by Dr. William Frazier, Pilgrim Consulting, LLC; Mr. Rick Russell, NASA; Dr. Yan Lu, NIST; Dr. Brandon D. Ribic, America Makes; and Caroline Vail, NSWC Carderock.

Joseph Pauza and Anthony Rollett, Materials Science and Engineering, Carnegie Mellon University, 5000 Forbes Ave, Pittsburgh, PA 15213. Contact e-mail: jpauza@andrew.cmu.edu. niques. The technique works in a layer-by-layer fashion to deposit material to build up a 3D metal part. First, a computeraided design (CAD) 3D model of a part is sliced into a stack of many layers. These layers are used as the blueprint for material deposition. In LPBF AM, the material is deposited via the melting of a powder layer using a laser. For each layer of the sliced CAD file, a thin layer of fine metal powder is deposited. A slice from the CAD file is overlaid on top of the powder layer, and the laser is used to melt the powder in the regions defined by the CAD slice. This technique allows for complex parts to be produced that cannot be easily manufactured via subtractive machining or casting. Parts with lattice structures or complex internal geometries are classic examples. The technology also lends itself to low-volume part production and rapid prototyping.

The technology has encountered a variety of challenges that have occupied a large portion of research efforts. Porosity stemming from keyholing (Ref 5, 6), lack of fusion between subsequent melt pool passes and layers (Ref 7), and residual porosity from the powder have been identified as serious issues with the manufacturing technique (Ref 8). Additionally, hot cracking during fabrication has been identified as a concern 
with regard to the successful fabrication of LPBF AM parts (Ref 9, 10). Significant progress has been made in addressing these defects.

One largely unrealized capability offered by LPBF and other AM techniques is that of microstructure control, which includes at least grain morphology, orientation, phase structure, and dislocation content. As microstructure directly impacts mechanical properties, microstructure control is of interest in virtually every manufacturing technique. For example, the columnar to equiaxed transition in casting has been a subject of study for many decades (Ref 11). LPBF AM is of interest in this respect due to the fact that a small melt pool is employed to manufacturing these parts. This means that the operators of a LPBF AM machine are making decisions about the part microstructure at a scale of order $100 \mu \mathrm{m}$. Accordingly, LPBF microstructures are complex and change dramatically depending on the build parameters employed to fabricate the parts (Ref 12).

Many reports on LPBF AM microstructure and microstructure control are already available (Ref 3, 13-16). Two important components of microstructure are grain morphology and crystallographic texture, and these are often referenced in many of these works. This work focuses on these two microstructural features and discusses the results with reference to relevant work.

Both Narra and Gockel investigated controlling prior-beta grain size in Ti64 parts using electron beam powder bed fusion (Ref 17, 18). They illustrated the use of constant melt pool cross section to drive constant beta grain size. Scaling the melt pool size scaled the prior-beta grain size in both full parts and thin walls. Dehoff et al. also investigated site-specific control of microstructure in an electron beam AM machine by varying parameters across different regions of the part (Ref 1). They focused on varying the solidification conditions to produce microstructures with less columnar character in specific regions. Popovich et al. used LPBF to produce functionally graded parts with different elastic moduli in different regions of the part (Ref 15). These studies illustrate the ability of the AM process to produce site-specific, controlled microstructures.

The previously mentioned studies clearly show that altering laser parameters in specific regions can be used to vary the associated microstructures. Despite the promising preliminary work cited above, full control over microstructures remains challenging. Clearly, this is a consequence of the complex nature of microstructure development in AM and the large number of processing parameters in AM. Geiger et al. showed how scan strategy can also drastically shift texture in IN738C LPBF parts (Ref 4). Changes in the rotation between layers and the rotation of the scan strategy with respect to the part can dramatically change the texture of the part. Andreau et al. studied texture development in SS316L produced via LPBF AM (Ref 14). The authors were able to make conclusions about the texture development by considering the overlap between subsequent melt pool passes and clearly observe the development of texture on the melt pool scale. This highlights the importance of considering melt pool interactions with previously deposited microstructure on the melt pool scale when considering AM texture and grain structure. Attard et al. investigated a variety of build parameters and their effect on grain structure and texture for a few scan strategies, including the Island scan strategy (Ref 12). They observed dramatic shifts in texture and grain morphology depending on the parameters selected. They then illustrated how this concept could be used in the fabrication of a turbine blade by imparting a desired microstructure onto the part in specific regions.

The complex nature of the microstructures and the large number of parameters that influence the final microstructure (power, velocity, hatch spacing, layer thickness, scan strategy, material, etc.) means that interpreting the microstructures from 2D cross sections after the build can be quite difficult. For example, there are frequent references to nucleation and equiaxed grain shape, but it is clear that, in the absence of serial sectioning, isolated $2 \mathrm{D}$ cross sections are not sufficient to make unambiguous claims about grain structure (Ref 19). Simulation work has shown that grains can appear equiaxed depending on the plane the cross section is made in, despite have a distinctly non-equiaxed shape (Ref 20).

Simulation studies of AM microstructure offer a partial solution. A complete understanding of the assumptions used to simulate microstructure development and the ability to evaluate full 3D synthetic microstructures can resolve confusion about the development of grain shapes and texture in LPBF AM parts. A variety of simulation methods have been employed to generate synthetic AM microstructures. Cellular automata have been frequently employed as a natural follow-on from their application to modeling solidification prior to the advent of metals AM (Ref 21-23). We recently showed that adding a misorientation dependent mobility function to a Monte Carlo Potts model enabled simulation of texture evolution in Inconel 718 parts fabricated via LPBF AM for a variety of laser parameters (Ref 20). This computationally efficient simulation approach offers the potential to further elucidate microstructure development in AM parts via computationally efficient production of bulk AM microstructures. However, as the simulation approach is still in its infancy, exploration of its predictive capabilities and limitations is still necessary.

We explore the effects of changing hatch spacing and layer thickness within the texture-aware solidification Potts model to understand the effect of these variables on the synthetic microstructures generated by this technique. We quantify both the grain morphology and crystallographic texture, as well as characterize the mechanical response of the microstructures via EVP-FFT micromechanical simulation. This study helps to understand what the modeling approach, in its current form, predicts the effect of hatch spacing and layer thickness alterations will have on microstructure development during LPBF AM builds. The conclusions reveal potentially necessary modifications to the textured solidification Potts model as point to potential strategies for microstructure control in LPBF AM parts.

\section{Methods}

The texture-aware solidification (TS) Potts model approach is derived from the classical grain growth Monte Carlo (MC) Potts model (Ref 24-26), and the use of Potts models for simulation of AM parts by Rodgers et al. (Ref 27, 28). The grain growth Potts model is an energy minimization approach that is able to accurately simulate curvature-driven grain growth (Ref 24). To visualize this simulation approach, consider a square 2D lattice of sites. Each site is assigned a spin value (grain ID), and each region of contiguous sites with the same spin value is considered a grain. At each timestep in a MC Potts model, grain growth simulation $n$ switch attempts are made, 
where $n$ is the total number of sites in the simulation volume. A switch attempt is when one site attempts to flip from one spin value to another. An energy calculation is made before and after the switch is made for a particular site $(\mathrm{Eq} 1) . E$ is the total system energy, $N N$ is the number of neighbors of the candidate site, $J$ is the Hamiltonian used to determine the energy between site $i\left(S_{\mathrm{i}}\right)$ and $j\left(S_{\mathrm{j}}\right)$, and $\delta$ is the Kronecker delta. This energy calculation can be understood to essentially be the number of unlike neighbors in the system. The more sites with neighbors of different spin, the higher the total system energy. Comparing the two energy calculations determines whether the switch would increase or decrease the system energy (Eq. 2). From there, the switch is either accepted or rejected via equation 3, where $W_{M}$ is the switching probability, $M$ is the switching mobility, $k_{\mathrm{B}}$ is the Boltzmann constant, and $T_{\text {sim }}$ is the simulation temperature. As more switches are accepted and the system evolves, the reduction in surface energy mimics grain growth. An important term in equation 3 is $M$. This term essentially acts as an additional control over the rate of switching in the simulation. In Eq. 3, $M$ can be described as the probability that a switch is accepted if it decreases the system energy. This term can be tuned to control the rate of coarsening in grain growth simulations.

$E=\sum_{i=1}^{n} \sum_{j=1}^{N N} \frac{J\left(S_{i} S_{j}\right)}{2}\left(1-\delta_{S_{i} S_{j}}\right)$

$\Delta E=E_{\text {final }}-E_{\text {initial }}$

$W_{M}=\left\{\begin{array}{l}M * \exp \left\{-\frac{\Delta E}{k_{B} T_{\text {sim }}}\right\} \text { if } \Delta E>0 \\ \text { Mif } \Delta E \leq 0\end{array}\right.$

In order to simulate AM processes, Rodgers et al. added a moving region of random sites to the simulation volume to simulate a melt pool (Ref 27). Additionally, a decaying mobility was employed around this simulated melt pool to create a heat-affected zone (HAZ). In a similar fashion, a moving heat source is added to the standard grain growth Potts model in order to simulate a melt pool moving through the synthetic volume. The Rosenthal solution is employed for its ease of implementation and computational efficiency (Ref 29). In Eq 4 and 5, $T$ is the physical temperature of the site, $T_{0}$ is the preheat temperature, $Q_{p}$ is the laser power, $\lambda$ is the absorptivity, $k$ is the thermal conductivity, $R$ is the radial position of the calculation site relative to the point heat source, $v$ is the laser velocity, $\alpha$ is the thermal diffusivity, $\xi$ is the position of the site in the direction of the point source movement, and $y$ and $z$ are the calculation site positions in the plane perpendicular to the point source movement direction.

The inclusion of this heat source transforms Eq. 3 into Eq. 6. $M(T)$ is a temperature-dependent mobility function as defined by Eq. 7. This allows the amount of grain growth to drop off as the temperature decreases as naturally occurs in the HAZ. For the simulations performed in this study, $p_{0}$ was set to $8.0 \times 10^{-25}$ and $p_{1}$ was set to 0.0347 . These values were selected in order to allow only small amounts of coarsening in the heat-affected zone.
$T=T_{o}+\frac{\lambda * Q_{p}}{2 \pi R k} e^{\frac{-v(\xi+R)}{2 \alpha}}$

$R=\sqrt{\xi^{2}+y^{2}+z^{2}}$

$W_{M}=\left\{\begin{array}{c}M(T) * \exp \left\{-\frac{\Delta E}{k_{B} T_{\text {sim }}}\right\} \text { if } \Delta E>0 \\ M(T) \text { if } \Delta E \leq 0\end{array}\right.$

$M(T)=p_{0} e^{\left(p_{1} * T\right)}$

In order to motivate solidification to occur in the simulated volume, all sites with temperature greater than $T_{m}$ (melting temperature) are assigned a spin value of 0 (Eq. 8. A stored energy value is then associated with this spin value (Eq. 9 such that the removal of these liquid sites is encouraged by minimization of total system energy (Eq. 10).

$I D=\left\{\begin{array}{l}\text { old state if } T \quad<T_{m} \\ 0 \text { if } T \geq T_{m}\end{array}\right.$

Stored Energy $=\left\{\begin{array}{c}0 \text { if } I D \\ E_{\text {stored }} \text { if ID }=0\end{array}>0\right.$

$\Delta E=E_{\text {final }}-E_{\text {initial }}+\Delta($ Stored Energy $)$

Simulation of solidification texture is accomplished by first assuming that material system being simulated solidifies as a phase with a cubic crystal structure. This allows for assumption of a $<001>$ preferred growth direction for the solidifying material. For sites switching from liquid (spin ID of 0 ) to solid, the switching mobility is assigned to be a function of the misorientation between the solidification direction and the nearest $<001>$ direction of the candidate solidification switch $(M(\theta))$. This causes Eq. 6 to become Eq. 11 .

$$
W_{M}=\left\{\begin{array}{cccc}
M(T) * \exp \left\{-\frac{\Delta E}{k_{B} T s_{\text {im }}}\right\} & \text { if } & \Delta E>0 & \text { and old state }>0 \\
M(T) & \text { if } & \Delta E \leq 0 & \text { and old state }>0 \\
M(\theta) * \exp \left\{-\frac{\Delta E}{k_{B} T_{\text {sim }}}\right\} & \text { if } & \Delta E>0 \text { and old state }=0 \\
M(\theta) & \text { if } & \Delta E \leq 0 \text { and old state }=0
\end{array}\right.
$$

The solidification direction $(\vec{G})$ is assumed to be the thermal gradient direction. Using the analytical Rosenthal solution, the thermal gradient direction can be quickly and easily calculated by taking the gradient of Eq. 4. In order to find the misorientation between this gradient direction and the nearest $<001>$ of a particular spin, first all spins must be assigned an orientation. This is done by assigning a set of three Euler angles to each spin value. From there, a transformation matrix (g) can be developed (Eq. 12) that can be used to calculate the $\langle 001\rangle$ directions of the particular orientation in the sample frame (Eq. 13). The misorientation between each of the six $<001>$ directions can then be easily calculated using Eq. 15, and the minimum is selected as the misorientation. 
$g=\left(\begin{array}{ccc}\cos \left(\phi_{1}\right) \cos \left(\phi_{2}\right)-\sin \left(\phi_{1}\right) \cos \left(\phi_{2}\right) & \sin \left(\phi_{1}\right) \cos \left(\phi_{2}\right)+\cos \left(\phi_{1}\right) \cos (\Phi) \sin \left(\phi_{2}\right) & \sin (\Phi) \sin \left(\phi_{2}\right) \\ -\cos \left(\phi_{1}\right) \sin \left(\phi_{2}\right)-\sin \left(\phi_{1}\right) \cos (\Phi) \cos \left(\phi_{2}\right) & -\sin \left(\phi_{1}\right) \sin \left(\phi_{2}\right)+\cos \left(\phi_{1}\right) \cos (\Phi) \sin \left(\phi_{2}\right) & \sin (\Phi) \cos \left(\phi_{2}\right) \\ \sin (\Phi) \sin \left(\phi_{1}\right) & -\sin (\Phi) \cos \left(\phi_{1}\right) & \cos (\Phi)\end{array}\right)$

$h^{\prime}=g^{T} h$

$\cos (\theta)=S D \cdot h^{\prime}$

Using this misorientation, the switching mobility can be determined using the function presented in Eq. 15. For the simulations performed in this study, $c_{0}$ and $c_{1}$ were set to 0.5 and $c_{2}$ was set to be 2.5. Previous work (Ref 20) had identified these values as giving the best fit between simulated and experimental microstructures

$M(\theta)=c_{0}+c_{1} \cos \left(c_{2} \theta\right)$

This function allows for orientations with $<001>$ directions that are well aligned with the solidification direction to grow more rapidly than other orientations based on the local switching events as liquid sites convert (transform) to solid. This approach has shown the ability to reproduce solidification textures observed in casting and LPBF AM applications (Ref 20).

Each simulation is initialized on a 3D lattice of cuboidal sites where each site is initially assigned a random spin. The melt pool is then allowed to move through the simulation domain at a constant step size in order to simulate the AM process. For these simulations, the voxel size was set to be 1 $\mu \mathrm{m} \times 1 \mu \mathrm{m} \times 1 \mu \mathrm{m}$ and each Monte Carlo step was set to be $10 \mu$ s. The set scan speed for the laser parameters used in the simulation was $1.2 \mathrm{~m} / \mathrm{s}$. At this scan speed, the melt pool can be assumed to have traveled $12 \mu \mathrm{m}$ or 12 voxels at each time step. At the end of each melt pool pass, once the heat source had reached a predefined position such that the melt pool completely passed through the simulated volume, the position of the melt pool in the layer was updated and the incremental movement of the melt pool was repeated. This updated position was defined by the preset hatch spacing for the simulation. The hatch spacing, as defined for this study, is the lateral offset for each melt pool within a single layer of melt pool passes. Once the total number of melt pool passes in one layer reaches the desired number, the layer height is updated and the process repeats itself. This updated layer height is defined by the assigned layer thickness. For ease of interpretation of the simulation results, no rotation or shift factor was employed for the current study.

For simulation of bulk AM microstructure, multiple passes and layers were simulated for each parameter set. Five melt pool passes and eight layers were simulated. The bulk microstructure was then extracted from each simulation. As mentioned previously, constant melt pool parameters were employed. These parameters calculate the temperature distribution at each time step via the Rosenthal solution (eq. 4). The parameters used were selected to approximate the constant material parameters of a Nickel-based superalloy. Values of the physical properties were assumed to have the following: thermal conductivity $(k) 11.2 \mathrm{Wm}^{-1} \mathrm{~K}^{-1}$, density $(\rho) 8220$ $\mathrm{kg} \cdot \mathrm{m}^{-3}$, specific heat $\left(C_{\mathrm{p}}\right) 650 \mathrm{~J} \cdot \mathrm{kg}^{-1} \mathrm{~K}^{-1}$, and melting temperature $\left(T_{m}\right) 1573 \mathrm{~K}$. Laser power $\left(Q_{\mathrm{p}}\right)$ was set to be $285 \mathrm{~W}$, velocity $(v) 1.2 \mathrm{~m} / \mathrm{s}$, and absorptivity $(\lambda) 0.155$.

To assess the effects of hatch spacing and layer thickness, 4 hatch spacings were investigated $(80 \mu \mathrm{m}, 90 \mu \mathrm{m}, 100 \mu \mathrm{m}$, and $110 \mu \mathrm{m})$ and 3 layer thicknesses were investigated at each of the hatch spacings $(30 \mu \mathrm{m}, 40 \mu \mathrm{m}$, and $50 \mu \mathrm{m})$. The bulk microstructure of each simulation measures $150 \mu \mathrm{m}$ in the scan direction, $400 \mu \mathrm{m}$ in the build direction, and between $320 \mu \mathrm{m}$ and $440 \mu \mathrm{m}$ in the transverse direction depending on the hatch spacing.

The model also assumes that no nucleation is occurring at the selected solidification parameters. Additionally, the model does not include undercooling in the calculation of the mobility, which can be considered analogous to solidification front velocity. This is accomplished by assuming large undercoolings and subsequently high solidification velocities such that the velocities of the dendrite tips are essentially equal to the melt pool velocity selected for the simulation.

\section{Results and Discussion}

\subsection{Synthetic Microstructure Results and Discussion}

The bulk microstructures as predicted by the TS Potts model simulation approach for each hatch spacing (HS) and layer thickness (LT) combination are presented in Fig. 1. These bulk microstructure simulations each took 7200 Monte Carlo steps to complete. They were run in parallel on 840 cores and each took between 140 and $150 \mathrm{cpu}$ hours to complete, depending on the size of the simulations.

A significant variation in microstructure morphology is evident. The low hatch spacing and low layer thickness microstructures exhibit large columnar grains with some grains spanning the height of the simulation volume. These results are consistent with experimental microstructures reported in literature. Andreau et al. reported microstructures in $316 \mathrm{~L}$ parts fabricated using the same, simplified scan strategy as was simulated in this work (Ref 14). They observe microstructures with large columnar grains that span hundreds of microns and possess the same $<101>/ /$ BD texture observed in Fig. 1. Additionally, Rowenhorst et al. generated a 3D reconstruction of AM microstructure via automated serial section EBSD that showed grains growing epitaxially through many layers of an AM build (Ref 30 ).

As hatch spacing and layer thickness increase, the columnar grains appear to break up and these smaller grains take on angular features that result from the partial remelting of grains by subsequent melt pool passes. Note that the HS100 LT50 and HS110 LT50 microstructures possess regions of lack of fusion 


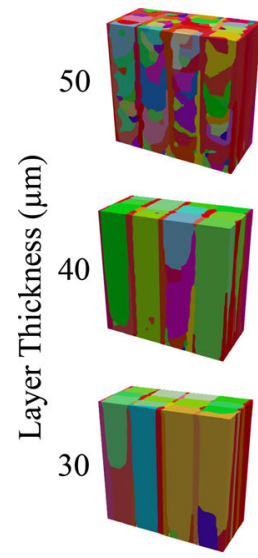

(a)
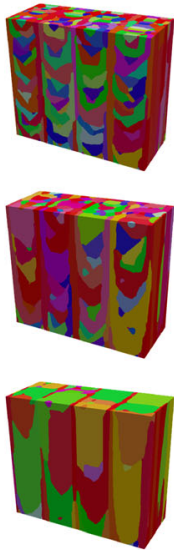

90
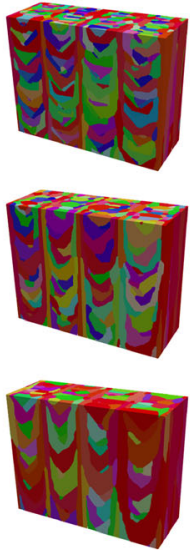

100
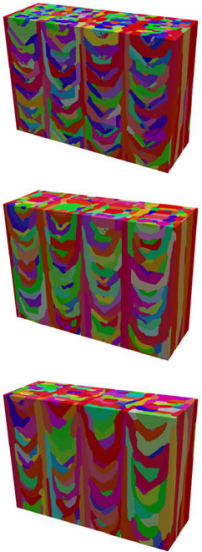

110

Hatch Spacing $(\mu \mathrm{m})$
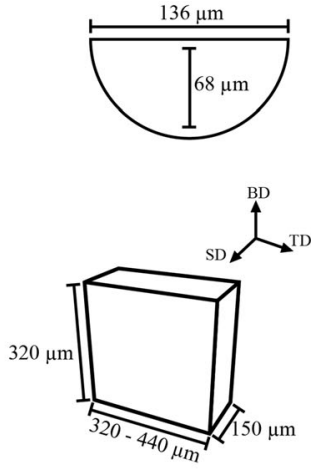

(b)

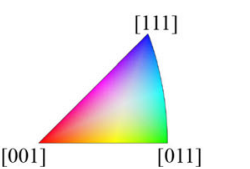

Fig. 1 Synthetic microstructures generated for each of the hatch spacing and layer thickness simulation cases (a). The power and scan speed are held constant, which means that the melt pool size is invariant (b). IPF coloring is with respect to the build direction (BD)

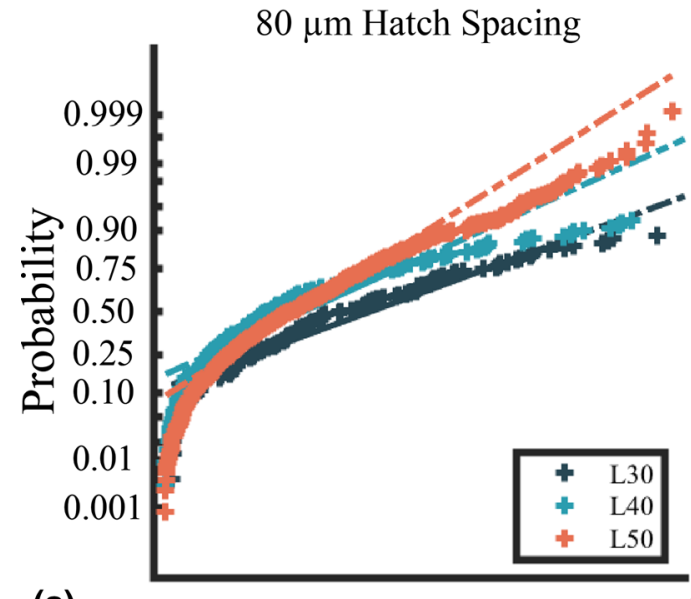

(a)

$100 \mu \mathrm{m}$ Hatch Spacing

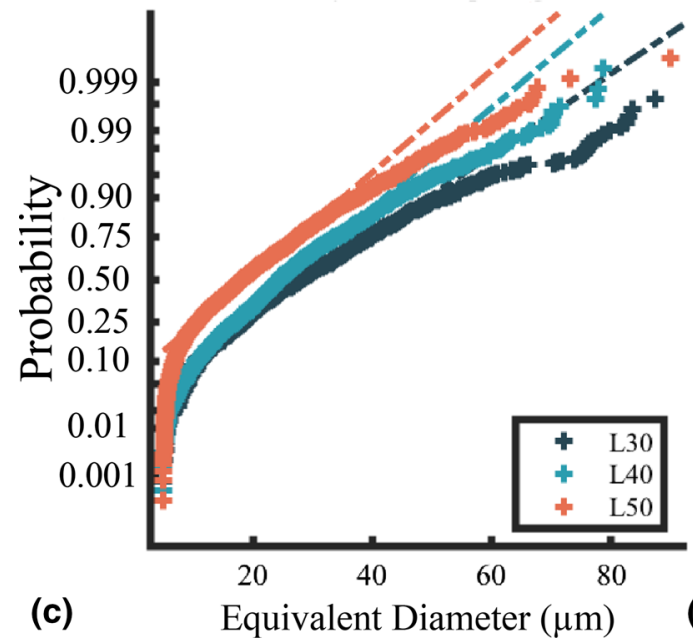

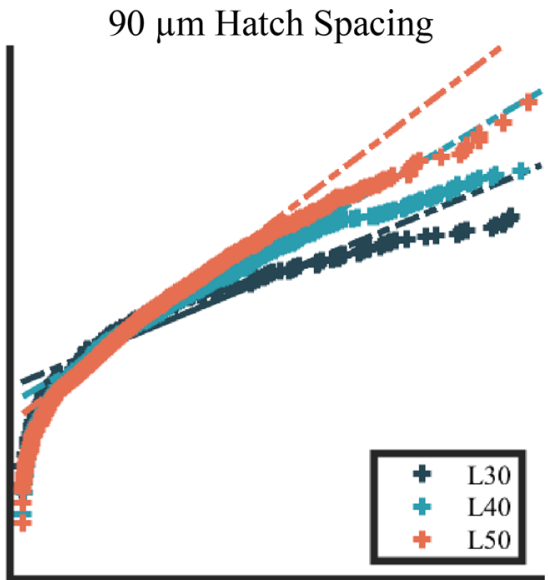

(b)

$110 \mu \mathrm{m}$ Hatch Spacing

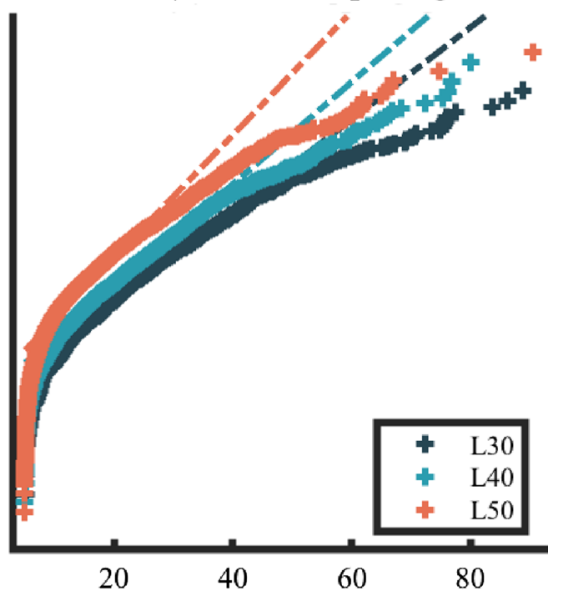

(d) Equivalent Diameter $(\mu \mathrm{m})$

Fig. 2 Probability paper plots of the equivalent diameter of each grain the synthetic microstructures. Normally distributed data falls on a straight line on such a plot. The upper tails deviate most strongly for the largest hatch spacing simulations. (a) Probability paper plots for each simulation case with $80 \mu \mathrm{m}$ hatch spacing. (b) Probability paper plots for each simulation case with $90 \mu \mathrm{m}$ hatch spacing. (c) Probability paper plots for each simulation case with $100 \mu \mathrm{m}$ hatch spacing. (d) Probability paper plots for each simulation case with $110 \mu \mathrm{m}$ hatch spacing 
(LOF) porosity. In this simulation approach, these regions are not voids but instead regions of randomized sites that remain unmelted and retain their original configuration from initialization of the simulation domain.

Qualitatively, a shift in grain size can be observed between the simulations. In order to quantify this, the equivalent diameter for each of the grains was calculated per simulation. These results are plotted on normal distribution plots in Fig. 2. From the results, it is obvious that none of the microstructures possess normal distributions of grain size as none of the curves lie on the straight line included on the plot. Additionally, the grain size decreases with increasing layer thickness within each hatch spacing. Less overlap in the simulations appears to result in less epitaxial, multilayer growth. Increased overlap between subsequent melt pool passes results in greater remelting, leaving less competition for the large columnar grains during solidification. For the simulations with less overlap, more of the fine grains that grow from the "powder layer" survive and prevent the epitaxial growth of the large columnar grains.

To assess texture evolution, the $<001>$ pole figure from each simulation case is plotted in Fig. 3. The pole figures were calculated using Dream3D (Ref 31). The three columnar microstructures (HS80 LT30, HS80 LT40, and HS890 LT30) possess very similar textures. This rotated cube texture is frequently reported in literature when LPBF AM builds have employed similar scan strategies (Ref 13, 14, 32, 33). As the grain structure shifts away from the columnar morphology, the texture also experiences a shift. Generally, as the amount of overlap between subsequent melt pool passes decreases, the texture strength decreases. Additionally, as the layer thickness decreases in the non-columnar microstructures, the simulations predict the development of a TD-direction fiber texture. While this texture has been previously reported for scan strategies similar to the one employed for the present study (Ref 20,34), this trend could be a by-product of the current texture development simulation approach. As mentioned previously, the simulation volume is initialized on a lattice of random spin values. These regions of randomized spins are the highest density of candidate orientations in the entire simulation volume. This means that the likelihood of a well-aligned spin value existing in these regions is much higher than in the regions of remelting where the microstructure is much coarser

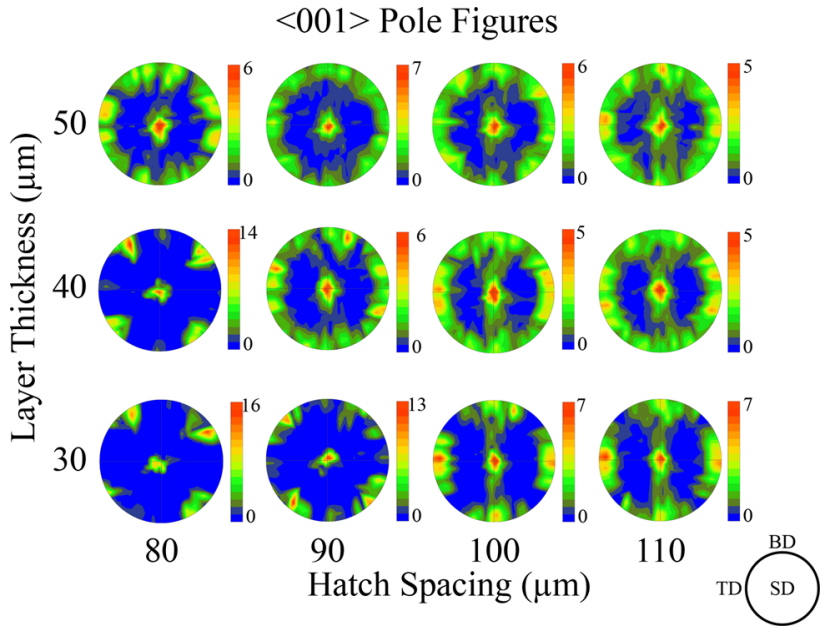

Fig. 3 The $<001>$ pole figures produced for each of the simulation cases and the number of different candidate spins is much lower. The simulation approach is likely artificially inflating the development of the TD fiber texture because, as the layer thickness decreases, the regions of the melt pool edges that are exposed to this high-density candidate spin region are the area of the melt pool where the thermal gradients point horizontally, or nearly horizontally. This effect could be potentially mitigated by coarsening the initial starting simulation domain or the inclusion of a small amount of nucleation to prevent this effect from dominating the texture development. As with all simulations, care should be taken when interpreting the texture prediction results, especially when experimental validation has not been performed.

Misorientation distributions were plotted for each simulation case to further quantify the crystallographic texture present in the synthetic microstructures. These distributions take into account the area of each grain boundary as calculations were made on a voxel-by-voxel basis. These misorientation distributions are plotted in Fig. 4. Again, a clear trend is visible. As the layer thickness increases, the distribution shifts toward the Mackenzie distribution (Ref 35). Additionally, bimodal distributions can be observed in a few of the simulation cases. This is likely associated with the large columnar $<101>/ / \mathrm{BD}$ grains possessing low misorientation between each other.

\subsection{Crystal Plasticity Results and Discussion}

To investigate the mechanical responses of the different microstructures, an elasto-viscoplastic (EVP) crystal plasticity model was employed to simulate the response of the synthetic microstructures under tensile loading. The fast Fourier transform (FFT) algorithm was implemented to solve to for the stress state with a 2D slice of each microstructure used as the input to the modeling approach (EVP-FFT). This image-based approach does not require meshing of the microstructure, and each voxel of the input microstructure is considered in the calculation, with the local properties of each voxel dependent, largely, on crystallographic orientation. Constant amounts of strain are applied at each time step, which stretches the voxels in the simulation grid. The modeling approach calculates the resulting stress and strain fields at each voxel as a result of this macroscopic strain. The calculation of the strain tensor at each voxel is presented in eq. 16 . In this equation, $\varepsilon(x)$ represents the strain tensor at the voxel of interest, $C$ is the elastic stiffness tensor, $\sigma$ is the stress, $\varepsilon^{p, t}$ is the total plastic strain at the previous time step, $\dot{\varepsilon}^{p}$ is the plastic strain rate at the current time step, and $\Delta \mathrm{t}$ is the size of the time step. The formulation for calculation of the plastic strain rate is given in eq. 7. In eq. 17, $\boldsymbol{m}^{s}(\boldsymbol{x})$ represents the Schmid tensor for slip system $s$ at $\boldsymbol{x}, \dot{\gamma}^{s}(\boldsymbol{x})$ is the shear rate of slip system $s, \dot{\gamma}_{o}$ is a normalization factor, $\tau^{s}(\boldsymbol{x})$ is the critically resolved shear stress (CRSS), and $n$ is the stress exponent. For additional details and derivations of the solution and the FFT-based methods implemented to solve for the finial stress and strain states, the reader is directed to the following manuscript (Ref 36). The Voce hardening law is incorporated into the model to simulate the changes in the critically resolved shear stress of the slip systems as shear strain accumulates (eq. 19) (Ref 37). In eq. $19 \tau_{0}^{s}$ is the initial CRSS, $\theta_{0}^{s}$ is the initial hardening rate, $\theta_{1}^{s}$ is the asymptotic hardening rata, $\tau_{0}^{s}+\tau_{1}^{s}$ is the back-extrapolated CRSS, and $\Gamma$ is the accumulated shear.

$\boldsymbol{\varepsilon}(\boldsymbol{x})=\boldsymbol{C}^{-1}(\boldsymbol{x}): \boldsymbol{\sigma}(\boldsymbol{x})+\boldsymbol{\varepsilon}^{p, t}(\boldsymbol{x})+\dot{\boldsymbol{\varepsilon}}^{p}(\boldsymbol{x}, \boldsymbol{\sigma}) \Delta \mathrm{t}$ 

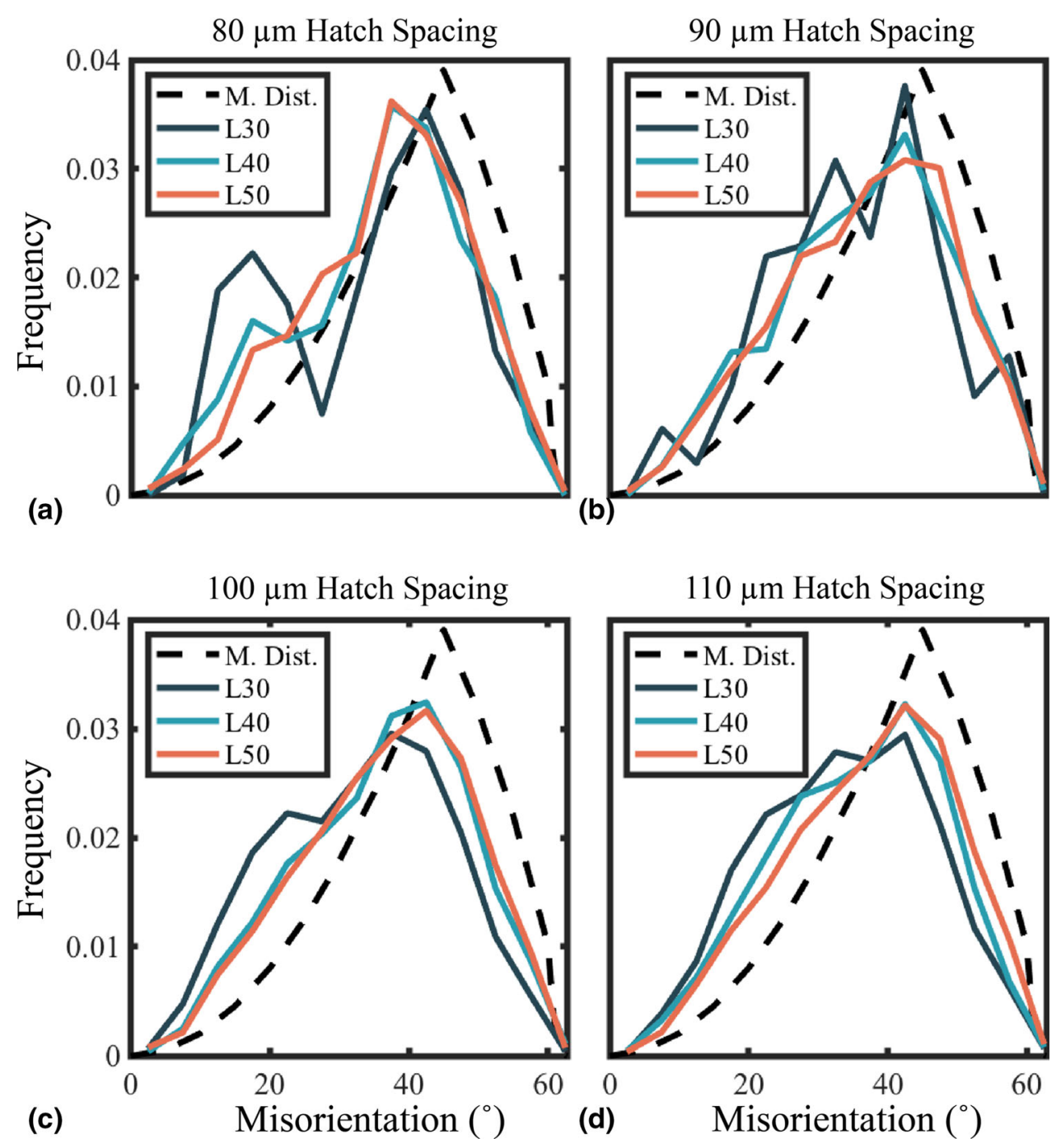

Fig. 4 Misorientation distributions for each simulation case. (a) Misorientation distributions for $80 \mu \mathrm{m}$ hatch spacing. (b) Misorientation distributions for $90 \mu \mathrm{m}$ hatch spacing. (c) Misorientation distributions for $100 \mu \mathrm{m}$ hatch spacing. (d) Misorientation distributions for $110 \mu \mathrm{m}$ hatch spacing

$$
\begin{aligned}
\dot{\boldsymbol{\varepsilon}}^{p}(\boldsymbol{x}, \boldsymbol{\sigma}) & =\sum_{s=1}^{N} \boldsymbol{m}^{\boldsymbol{s}}(\boldsymbol{x}) \dot{\gamma}^{s}(\boldsymbol{x}) \\
& =\dot{\gamma}_{o} \sum_{s=1}^{N} \boldsymbol{m}^{s}(\boldsymbol{x})\left(\frac{\left|\boldsymbol{m}^{\boldsymbol{s}}(\boldsymbol{x}): \boldsymbol{\sigma}(\boldsymbol{x})\right|}{\tau^{s}(\boldsymbol{x})}\right)^{n} \operatorname{sgn}\left(\boldsymbol{m}^{\boldsymbol{s}}(\boldsymbol{x}): \boldsymbol{\sigma}(\boldsymbol{x})\right)
\end{aligned}
$$

$\tau^{s}(\Gamma)=\tau_{0}^{s}+\left(\tau_{1}^{s}+\theta_{1}^{s} \Gamma\right)\left(1-\exp \left(-\Gamma\left|\frac{\theta_{0}^{s}}{\tau_{1}^{s}}\right|\right)\right)$

The stress-strain curves for each of the different microstructures for loading in both the build direction (BD) and transverse direction (TD) are presented in Fig. 5. 2D slices taken from the center of the microstructures were used as inputs to the mechanical model. Inconel 625 material parameters were used in keeping with the approach of approximating a Nickel-based superalloy material system (Ref 38). These elastic stiffness constants are presented in Table 1 . The Voce hardening law parameters that were used are shown in Table 2.
For easier interpretation of the results, the elastic modulus, yield stress, and anisotropy $\left(\sigma_{y}^{B D} / \sigma_{y}^{T D}\right)$ are plotted in Fig. 6 in a grid format based on the variation in hatch spacing and layer thickness. Elastic modulus was measured from the linear portion of each plot, and yield stress was determined via the $0.2 \%$ offset approach. Additionally, the multiples of random values extracted from inverse pole figures plotted with respect to the $\mathrm{BD}$ and $\mathrm{TD}$ were used to compare the intensity of $<001>$ crystallographic planes parallel to the $\mathrm{BD}$ and $\mathrm{TD}$ directions (Fig. 6f).

Some trends are evident. The most obvious trend is the BDdirection yield strength increases as the layer thickness and hatch spacing increases. Additionally, the anisotropy observed in the simulations appears to increase as the hatch spacing and layer thickness increase. These effects appear to be explained by the texture shifts as illustrated in Fig. 6(f). The anisotropy measured in Fig. 6(e) is the ratio of the BD-direction yield stress to the TD-direction yield stress. The large hatch spacing and layer thickness cases possess high BD-direction yield stress and low TD-direction yield stresses, resulting in anisotropy measures greater than 1 . Each simulation case with a yield 

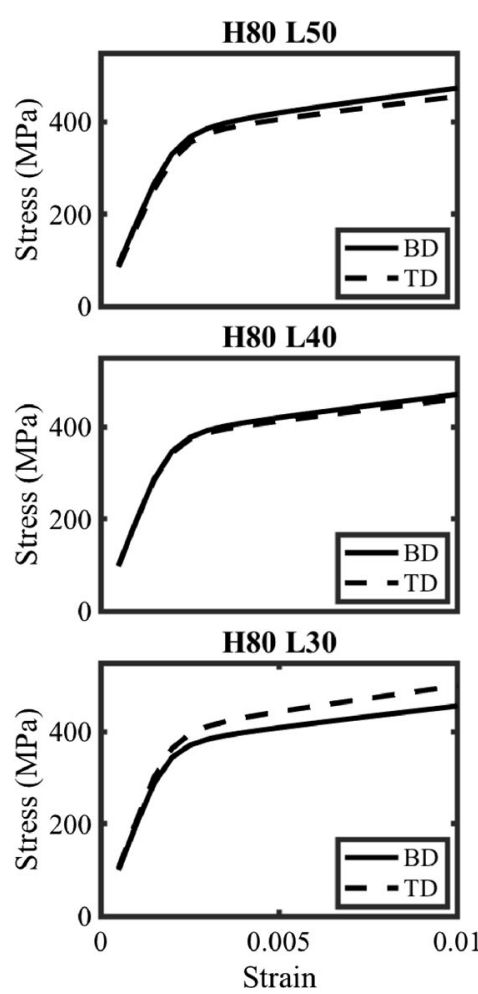

H90 L50

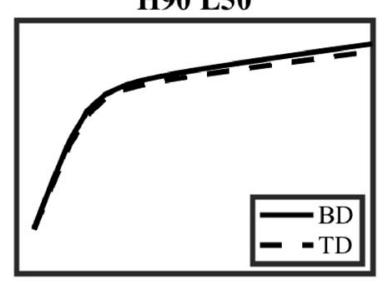

H90 L40

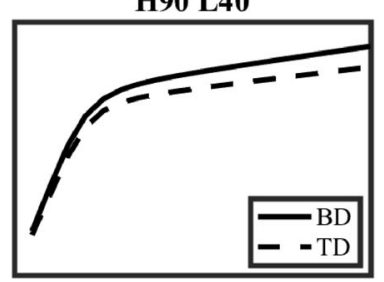

H90 L30

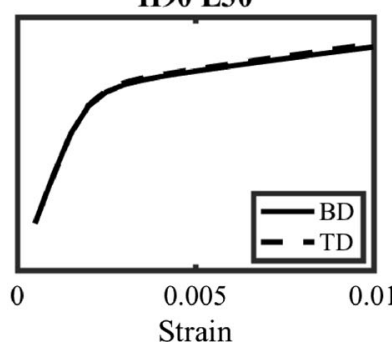

H100 L50

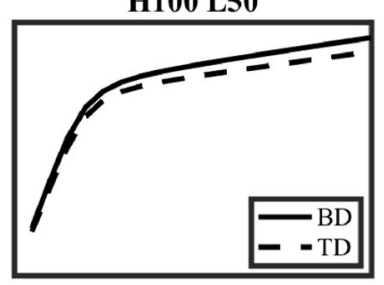

H100 L40

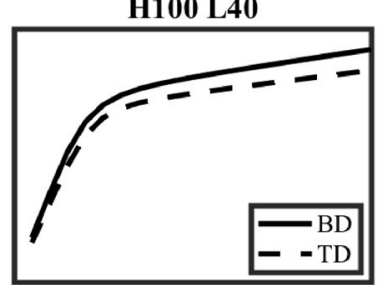

H100 L30

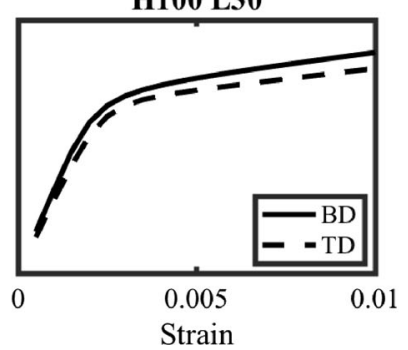

H110 L50

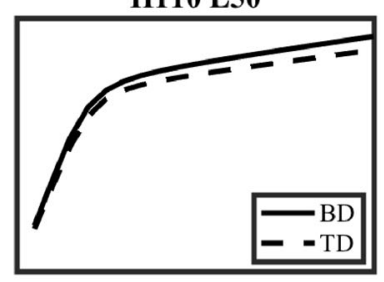

H110 L40

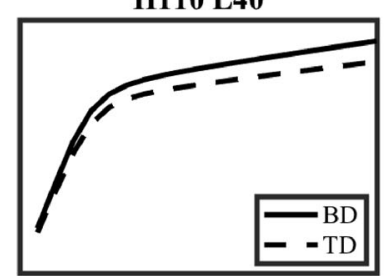

H110 L30

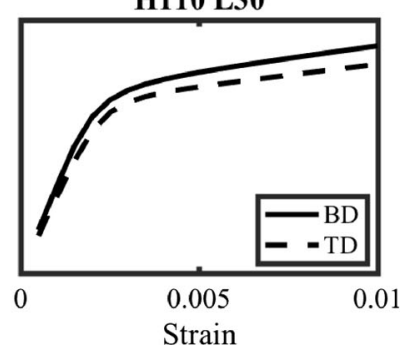

Fig. 5 Simulated stress-strain curves for each Synthetic microstructure. Curves are plotted for tension in both the build direction (BD) and transverse direction (TD).

Table 1 Single crystal elastic stiffness constants (GPa)

\begin{tabular}{lccc}
\hline Material & $\mathbf{C}_{\mathbf{1 1}}$ & $\mathbf{C}_{\mathbf{1 2}}$ & $\mathbf{C}_{\mathbf{4 4}}$ \\
\hline Inconel 625 & 243. & 156.7 & 117.8 \\
\hline
\end{tabular}

Table 2 Voce Hardening Law parameters

\begin{tabular}{lccc}
\hline$\tau_{\mathbf{0}}^{s}$, MPa & $\tau_{\mathbf{1}}^{s}, \mathbf{M P a}$ & $\theta_{\mathbf{0}}^{s}$ & $\theta_{\mathbf{1}}^{\text {s }}$ \\
\hline 165 & 400 & 1800 & 140 \\
\hline
\end{tabular}

stress anisotropy greater than 1 possesses low values as plotted in Fig. 6(f), indicating that the microstructures possess more $<001>$ crystal directions parallel with the TD than the BD. As the $<001>$ direction is the soft direction in cubic materials, the larger concertation of the $<001>$ crystal directions results in a lower yield stress for the microstructures in the TD than the BD. This is most clearly observed in the HS80 LT30 case and the HS90 LT30 case. These microstructures are the only ones which show a yield stress ratio of less than 1 . This result is nicely coupled with the large ratio for each microstructure shown in Fig 6(f). The large amount of $<001>$ crystal directions parallel to the $\mathrm{BD}$ in these simulation cases was responsible for the measured drop in build direction yield strength. These results indicate the large effect the texture shifts can have on the mechanical properties of the printed material.

\subsection{Quantification of Grain Morphology}

The grains in LPBF AM parts vary greatly in size and morphology. Measuring only the size of the grains neglects much of the information present in the microstructure. Qualitatively, from Fig. 1, a significant shift in the morphology of the grains present in the microstructure can be observed such that the most columnar shapes when large overlap between subsequent melt pool passes is employed. In order to further quantify the microstructures, analysis of the shapes present in the microstructure was performed via the use of second-order moment invariants. Moments invariants have been used in pattern recognition since $\mathrm{Hu}$ first proposed the approach in 1962 (Ref 39). Additionally, the use of moment invariants as measure of shape has already been successfully implemented in the study of microstructure. MacSleyne et al., for example, used 2-D moment invariants to quantify the evolution of $\gamma^{\prime}$ precipitate morphology throughout a 2-D phase field simulation (Ref 40).

In a similar fashion, 2-D moment invariants are used to quantify the grain shapes present in 2-D slices of each of our simulated microstructures. For these voxelized microstructures, equation 19 is employed to calculate the 2-D moment (of order $\mathrm{p}+\mathrm{q}$ ) for a particular grain ID. In this equation, $x$ and $y$ are the Cartesian coordinates of a particular voxel and $f(x, y)$ is an indicator function where $f(x, y)$ is equal to one when the grain ID of the voxel is equal to the grain ID of interest and zero when the grain ID of the voxel is different from the grain ID of interest.

In order to make these moments invariant to features of the image like position, size, and rotation, they can be transformed into moment invariants. These moment invariants are better suited to function as pure shape descriptors of the grains in the 


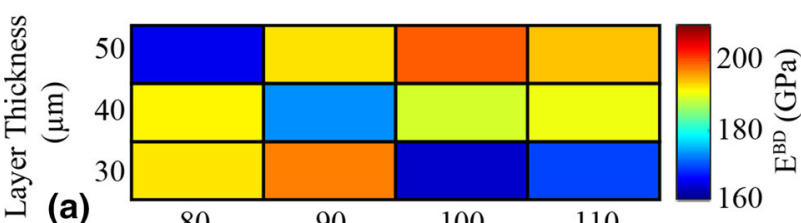

(a)

$\begin{array}{llll}80 & 90 & 100 & 110\end{array}$
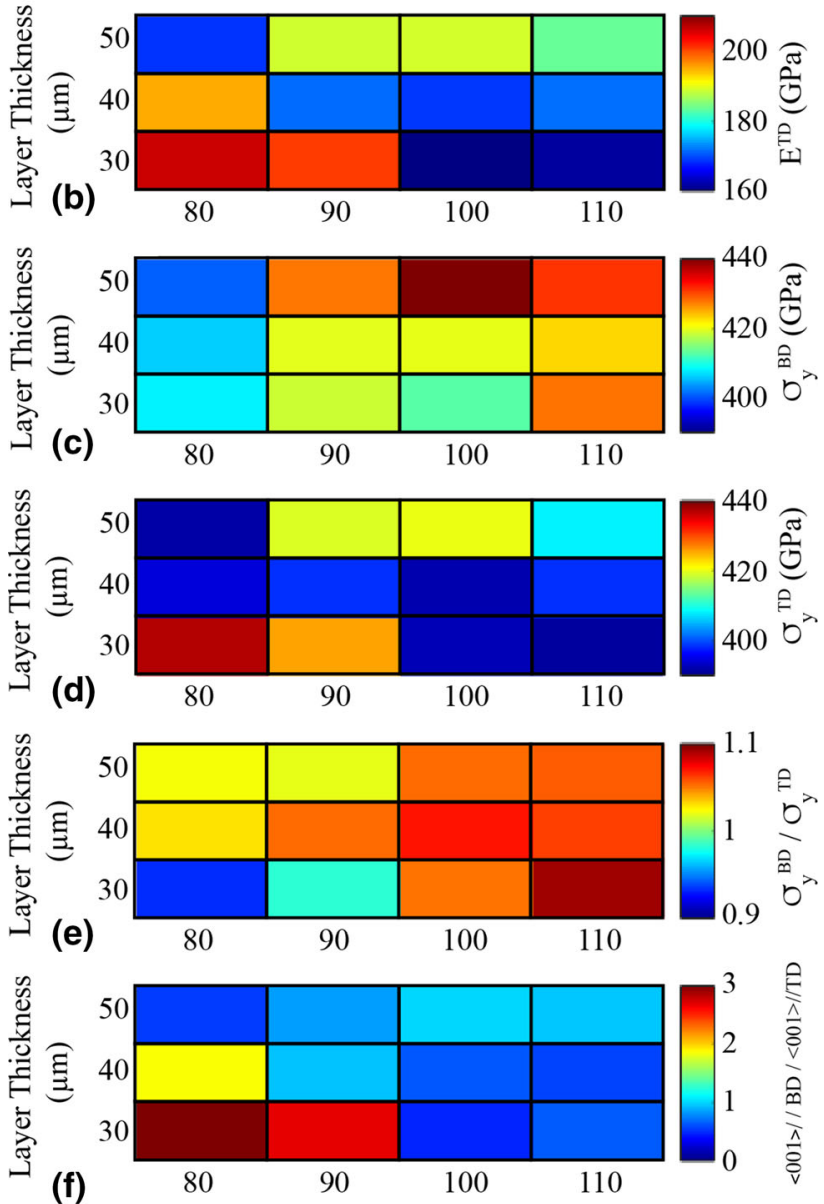

Hatch Spacing $(\mu \mathrm{m})$

Fig. 6 Gridded plots of (a) modulus in the build direction, (b) modulus in the transverse direction, (c) yield stress in the build direction, (d) yield stress in the transverse direction, (e) anisotropy between the yield stress in the build direction and transverse direction $\left(\sigma_{\mathrm{y}}^{\mathrm{BD}} / \sigma_{\mathrm{y}}^{\mathrm{TD}}\right)$, and (f) the ratio of the intensity of $<001>$ crystal planes parallel to the $\mathrm{BD}$ and to the $\mathrm{TD}$

microstructure. Equations 20 and 21 are the moment invariants calculated to quantify the shapes of each grain of each microstructure slice. In these equations, $A$ is the area of each grain $\left(M_{00}\right)$.

$M_{p q}=\sum_{x} \sum_{y} x^{p} y^{q} f(x, y)$

$\omega_{1}=\frac{A^{2}}{M_{20}+M_{02}}$

$\omega_{2}=\frac{A^{2}}{M_{20} M_{02}-M_{11}^{2}}$

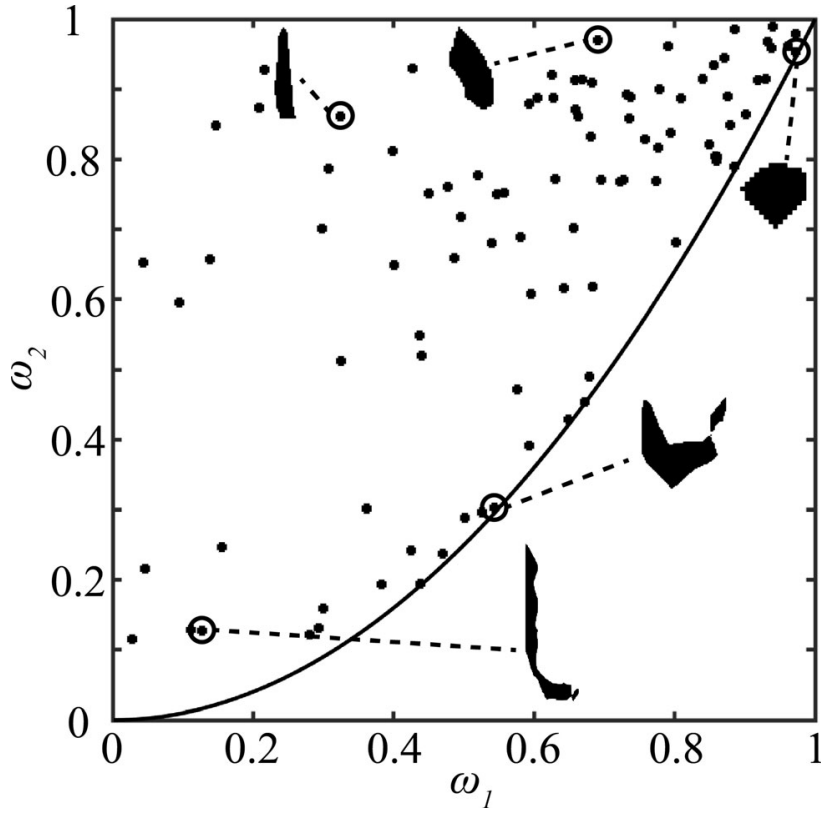

Fig. 7 Second-order moment invariant map for the H80 L50 microstructure. Selected grains are highlighted to illustrate their locations on the map

Figure 7 illustrates the second-order moment invariant map (SOMIM) for the HS80 L50 microstructure. A few selected grains have been highlighted on the map to illustrate where different shapes appear on the SOMIM. Nearly circular grains are located in the top right of the SOMIM, whereas elongated shapes are plotted in the top left. The $\omega_{1}$ axis is essentially a measure of aspect ratio, and the $\omega_{2}$ axis can be described as a measure of shape complexity.

Figure 8 presents the SOMIM for each of the microstructures, which shows that each microstructure has a distinctive fingerprint. The SOMIMs for columnar microstructures (HS80 LT30, HS80 LT40, and HS90 LT40) are concentrated near the top of the map, i.e., $\omega_{2} \approx 1$. As the hatch spacing and layer thickness increases, the density of near-circular grains increases, i.e., the points concentrate in the top right corner. Additionally, there many grains close to the right bound of the SOMIM. These correspond to an approximately triangular shape with angular features that appear in many of the microstructures. The SOMIM maps thus quantify the shifts in grain morphology found in the dataset that are also evident by inspection.

In order to link these variations to properties, each point was colored with respect to the average stress measured across each grain at the final EVP-FFT simulation step. Figure 8 illustrates the average stress response for BD loading. Clear clustering of some colors can be observed, indicating that placement on the map can be correlated, to some extent, with mechanical response. It appears that grains with low $\omega_{1}$ values appear to possess low average stress in the BD-direction loading case. These grains correspond to the thin, high aspect ratio grains that run along the centerline of the melt pools through multiple layers. These grains possess an $<001>$ direction parallel to the build direction, resulting in the low average stress value observed.

While the mechanical response of these grains is largely dependent on crystallographic orientation in the utilized EVPFFT simulation approach, the information presented here is still useful. Clearly the grain shapes developed in these microstruc- 

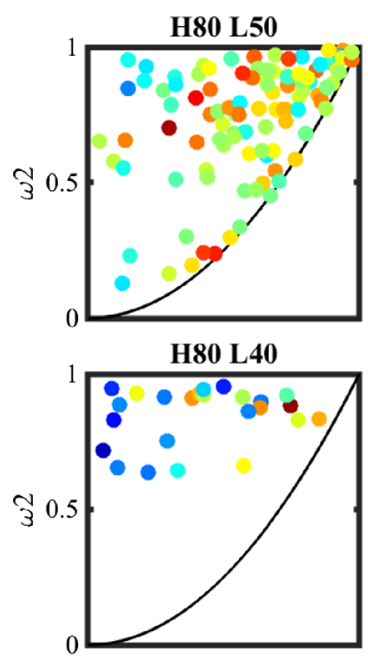

H80 L30

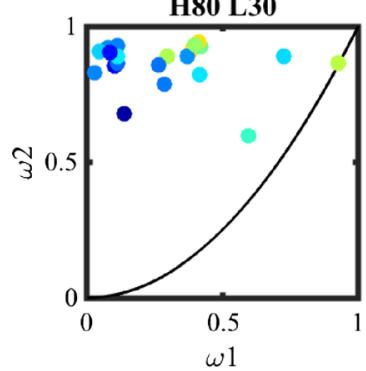

H90 L50

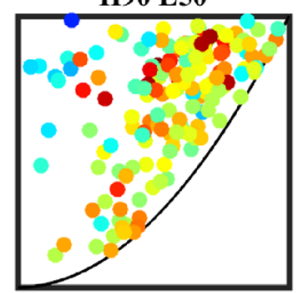

H90 L40

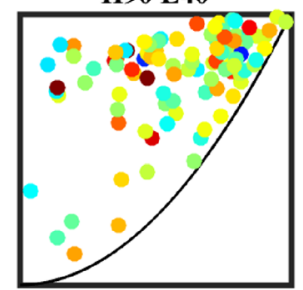

H90 L30

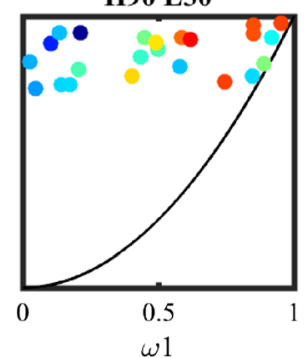

H100 L50

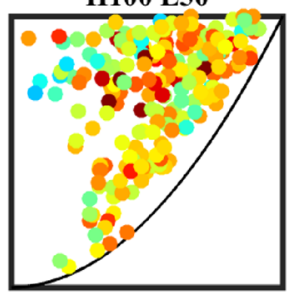

H100 L40

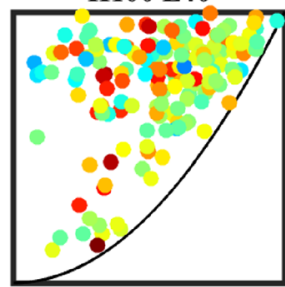

H100 L30

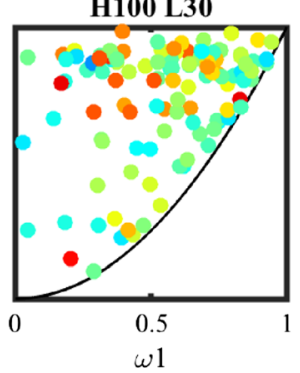

H110 L50

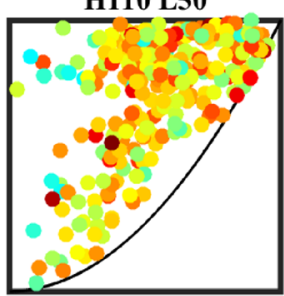

H110 L40

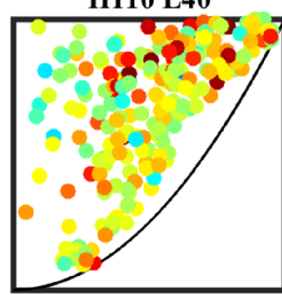

H110 L30

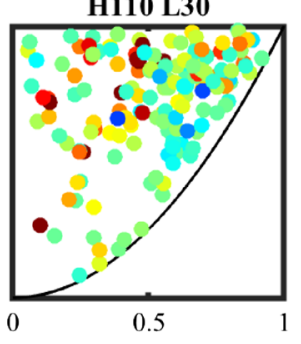

$\omega 1$
700

650

600

550

400

350

300

250

Fig. 8 Second-order moment invariant maps for each of the simulated microstructures, showing that the most columnar microstructures have points close to $\mathrm{w}_{2} \approx 1$ (top left) whereas the more complex microstructures concentrate along the right (curved) boundary of the space and the more circular grains concentrate at around $\mathrm{w}_{1} \approx 1$ and $\mathrm{w}_{2} \approx 1$ (top right). Points are colored by average stress $\left(\sigma_{33}\right)$ at final step of EVP-FFT simulation with BD loading direction

tures are coupled to the crystallographic orientation and, as a result, the mechanical response. This is because preferred solidification is present in the simulation approach and the shape and position of the grains is linked to the complex thermal history and changing solidification directions inherent to the manufacturing technique. These results indicate promise in use of the SOMIM plots to evaluate microstructure by linking grain shapes and properties in AM parts.

\subsection{Simulation of a Functionally Graded Microstructure}

As these microstructures clearly show strong effects on texture, grain shape, and mechanical response from the changes in hatch spacing and layer thickness, the ability to create functionally graded microstructures from altering these parameters becomes a possibility. In order to investigate the feasibility of this approach within the texture-aware Potts model, a simulation was run with the same parameters as previously outlined but with a shift in layer thickness midbuild. The majority of the simulation imposes a $40 \mu \mathrm{m}$ layer thickness. For 8 layers mid-build, the layer thickness was increased to $50 \mu \mathrm{m}$. The synthetic microstructure generated from this approach is illustrated in Fig. 9. A clear shift in microstructure can be observed where the layer thickness is shifted mid-simulation.

The graded microstructure presented in Fig. 9 was also subject to the micromechanical EVP-FFT simulations. Again, loading in both the BD and TD was simulated. Overall there was minimal anisotropy in the bulk mechanical response of the microstructure. However, when looking at the spatially resolved stress states from the different loading conditions as presented in Fig. 9(b) and Fig. 9(c), the effect of the microstructure shift can be observed. In the TD, the $50-\mu \mathrm{m}$ LT zone shows a significantly smaller stress response in than the $40-\mu \mathrm{m}$ zone. The SOMIM plots for both the BD and TD loading are presented in Fig. 9(d) and (e), respectively. Again, the cluster of low stress grains at low $\omega_{1}$ values is present. When plotted for the TD loading simulation, these grains possess much higher stress response. This indicates the high level of anisotropy generated in these builds. Precise control of microstructure for reduction in stress near potential stress concentrations could be accomplished by shift in microstructure via hatch spacing and layer thickness modifications.

\section{Conclusions}

- Systematic variation of the hatch spacing and layer thickness in the TS Potts simulations of LPBF AM microstructures reveals significant variation in the synthetic microstructures produced.

- The texture predicted by the approach also shows significant shifts across HS-LT space.

- The texture shift into the TD-direction fiber texture is an artifact of the current simulation approach. The region of random spin values used to initialize the synthetic volume 

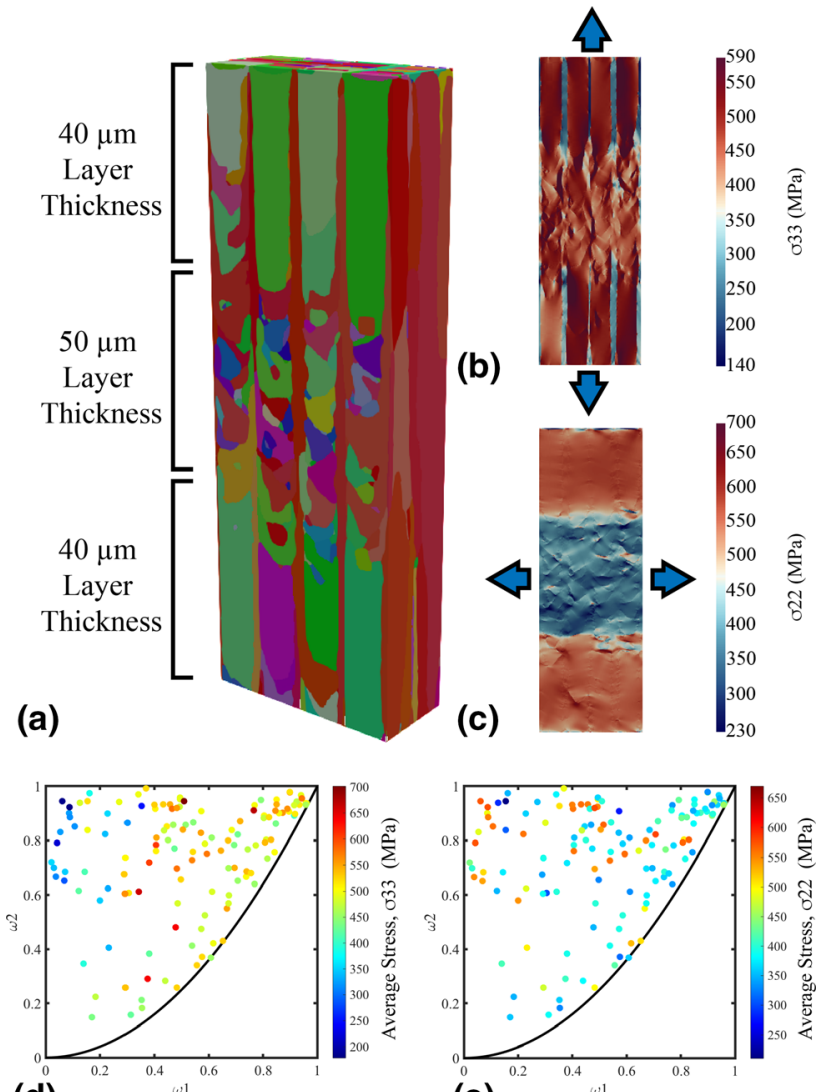

(d)

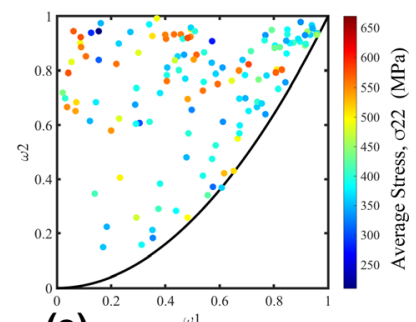

(e)

Fig. 9 Graded microstructure simulated by altering hatch spacing mid-simulation. (a) Synthetic graded microstructure. (b) Stress response of the microstructure during simulation of BD-direction tension. (c) Stress response of the microstructure during simulation of TD-direction tension. (d) SOMIM colored by average stress during simulated BD-direction loading. (e) SOMIM colored by average stress during simulated TD-direction loading

and simulate the powder layer artificially exaggerates the texture strength in regions of the melt pool that interact with these powder regions. Modifications to the modeling approach may be necessary to mitigate this effect and better reproduce the textures present in experimental LPBF AM builds.

- Scan strategies that extensively overlap successive melt pool passes (i.e., substantial re-melt) in the TS Potts approach result in synthetic microstructures with large columnar grains with strong $<101>/ / \mathrm{BD}$ texture when no melt pool shift or rotation between layers is present. As the overlap decreases, grain size also decreases.

- A distinctly non-random misorientation distribution was observed for nearly all synthetic microstructures generated. This included a distinct bimodal distribution for the HS 80 LT 30 microstructure. Less overlap resulted in a shift toward the Mackenzie distribution.

- Classification of the grain morphology through the use of second-order moment invariants quantified the variations in shape (grain morphology) of the microstructures. Different fingerprints for each microstructure were developed that resembled qualitative observations of grain morphologies present across the synthetic microstructures.

- The variations in microstructure were shown to have strong effects on the mechanical response as predicted by an EVP-FFT micromechanical model. The yield strength increased as the material shifted away from the strong $<101>$ textures of the columnar microstructures.

- Stress development in the individual grains could be correlated with grain shape when plotted on the second-order moment invariant map. This highlights the potential for grain shape to be linked to properties when analyzing AM microstructures.

- A functionally graded synthetic microstructure was simulated by altering the layer thickness of the build mid-simulation. Significant differences in mechanical response were evident ( $\sim 150 \mathrm{MPa}$ of stress) between the two regions of microstructure.

\section{Acknowledgments}

Resources supporting this work were provided by the NASA HighEnd Computing (HEC) Program through the NASA Advanced Supercomputing (NAS) Division at Ames Research Center.

\section{Open Access}

This article is licensed under a Creative Commons Attribution 4.0 International License, which permits use, sharing, adaptation, distribution and reproduction in any medium or format, as long as you give appropriate credit to the original author(s) and the source, provide a link to the Creative Commons licence, and indicate if changes were made. The images or other third party material in this article are included in the article's Creative Commons licence, unless indicated otherwise in a credit line to the material. If material is not included in the article's Creative Commons licence and your intended use is not permitted by statutory regulation or exceeds the permitted use, you will need to obtain permission directly from the copyright holder. To view a copy of this licence, visit http://creativecommons.org/licenses/by/4.0/.

\section{References}

1. R.R. Dehoff, M.M. Kirka, W.J. Sames, H. Bilheux, A.S. Tremsin, L.E. Lowe and S.S. Babu, Site Specific Control of Crystallographic Grain Orientation Through Electron Beam Additive Manufacturing, Mater. Sci. Technol., 2014, 31(8), p 931-938.

2. R. Shi, S.A. Khairallah, T.T. Roehling, T.W. Heo, J.T. McKeown and M.J. Matthews, Microstructural Control in Metal Laser Powder Bed Fusion Additive Manufacturing Using Laser Beam Shaping Strategy, Acta Mater, 2020, 184, p 284-305. https://doi.org/10.1016/j.actamat. 2019.11.053

3. N. Nadammal, S. Cabeza, T. Mishurova, T. Thiede, A. Kromm, C. Seyfert, L. Farahbod, C. Haberland, J.A. Schneider, P.D. Portella and G. Bruno, Effect of Hatch Length on the Development of Microstructure, Texture and Residual Stresses in Selective Laser Melted Superalloy Inconel 718, Mater. Des., 2017, 134, p 139-150. https://d oi.org/10.1016/j.matdes.2017.08.049

4. F. Geiger, K. Kunze and T. Etter, Tailoring the Texture of IN738LC Processed by Selective Laser Melting (SLM) by Specific Scanning Strategies, Mater. Sci. Eng. A, 2016, 661, p 240-246. https://doi.org/10. 1016/j.msea.2016.03.036

5. W.E. King, H.D. Barth, V.M. Castillo, G.F. Gallegos, J.W. Gibbs, D.E. Hahn, C. Kamath and A.M. Rubenchik, Observation of Keyhole-Mode Laser Melting in Laser Powder-Bed Fusion Additive Manufacturing, $J$. Mater. Process. Technol., 2014, 214(12), p 2915-2925. https://doi.org/ 10.1016/j.jmatprotec.2014.06.005 
6. R. Cunningham, C. Zhao, N. Parab, C. Kantzos, J. Pauza, K. Fezzaa, T. Sun and A.D. Rollett, Keyhole Threshold and Morphology in Laser Melting Revealed by Ultrahigh-Speed x-Ray Imaging, Science, 2019, 363(6429), p 849-852. https://doi.org/10.1126/science.aav4687

7. M. Tang, P.C. Pistorius and J.L. Beuth, Prediction of Lack-of-Fusion Porosity for Powder Bed Fusion, Addit. Manuf., 2017, 14, p 39-48. h ttps://doi.org/10.1016/j.addma.2016.12.001

8. R. Cunningham, S.P. Narra, T. Ozturk, J. Beuth and A.D. Rollett, Evaluating the Effect of Processing Parameters on Porosity in Electron Beam Melted Ti-6Al-4V via Synchrotron X-Ray Microtomography, Jom, 2016, 68(3), p 765-771.

9. E. Chauvet, P. Kontis, E.A. Jägle, B. Gault, D. Raabe, C. Tassin, J.-J. Blandin, R. Dendievel, B. Vayre, S. Abed and G. Martin, Hot Cracking Mechanism Affecting a Non-Weldable Ni-Based Superalloy Produced by Selective Electron Beam Melting, Acta Mater., 2018, 142, p 82-94. https://doi.org/10.1016/j.actamat.2017.09.047

10. P.-J. Chiang, R. Jiang, R. Cunningham, N. Parab, C. Zhao, K. Fezzaa, T. Sun and A.D. Rollett, In situ characterization of hot cracking using dynamic X-ray radiography, Advanced real time imaging II. J. Nakano, P.C. Pistorius, C. Tamerler, H. Yasuda, Z. Zhang, N. Dogan, W. Wang, N. Saito, B. Webler Ed., Springer International Publishing, Cham, 2019

11. J.D. Hunt, Steady State Columnar and Equiaxed Growth of Dendrites and Eutectic, Mater. Sci. Eng., 1984, 65(1), p 75-83. https://doi.org/10. 1016/0025-5416(84)90201-5

12. B. Attard, S. Cruchley, C. Beetz, M. Megahed, Y.L. Chiu and M.M. Attallah, Microstructural Control during Laser Powder Fusion to Create Graded Microstructure Ni-Superalloy Components, Addit. Manuf., 2020, 36, 101432. https://doi.org/10.1016/j.addma.2020.101432

13. G.E. Bean, T.D. McLouth, D.B. Witkin, S.D. Sitzman, P.M. Adams and R.J. Zaldivar, Build Orientation Effects on Texture and Mechanical Properties of Selective Laser Melting Inconel 718, J. Mater. Eng. Perform., 2019, 28(4), p 1942-1949. https://doi.org/10.1007/s11665019-03980-w

14. O. Andreau, I. Koutiri, P. Peyre, J.-D. Penot, N. Saintier, E. Pessard, T. De Terris, C. Dupuy and T. Baudin, Texture Control of 316L Parts by Modulation of the Melt Pool Morphology in Selective Laser Melting, $J$. Mater. Process. Technol., 2019, 264, p 21-31. https://doi.org/10.1016/j. jmatprotec.2018.08.049

15. V.A. Popovich, E.V. Borisov, A.A. Popovich, V.S. Sufiiarov, D.V. Masaylo and L. Alzina, Functionally Graded Inconel 718 Processed by Additive Manufacturing: Crystallographic Texture, Anisotropy of Microstructure and Mechanical Properties Functionally Graded Inconel 718 Processed by Additive Manufacturing: Crystallographic Texture, Anisot, JMADE, 2016, 114(November), p 441-449. https://doi.org/10. 1016/j.matdes.2016.10.075

16. L. Thijs, F. Verhaeghe, T. Craeghs, J. Van Humbeeck and J.P. Kruth, A Study of the Microstructural Evolution during Selective Laser Melting of Ti-6Al-4V, Acta Mater., 2010, 58(9), p 3303-3312. https://doi.org/ 10.1016/j.actamat.2010.02.004

17. S.P. Narra, R. Cunningham, J. Beuth and A.D. Rollett, Location Specific Solidification Microstructure Control in Electron Beam Melting of Ti-6Al-4V, Addit. Manuf., 2018, 19, p 160-166. https://d oi.org/10.1016/j.addma.2017.10.003

18. J. Gockel, J. Fox, J. Beuth and R. Hafley, Integrated Melt Pool and Microstructure Control for Ti-6Al-4V Thin Wall Additive Manufacturing, Mater. Sci. Technol., 2015, 31(8), p 912-916. https://doi.org/10. 1179/1743284714Y.0000000704

19. M.V. Kral and G. Spanos, Three Dimensional Morphology of Cementite Precipitates, Scr. Mater., 1997, 36(8), p 875.

20. J.G. Pauza, W.A. Tayon and A.D. Rollett, Computer Simulation of Microstructure Development in Powder-Bed Additive Manufacturing with Crystallographic Texture, Model. Simul. Mater. Sci. Eng., 2021, 29, p 055019.

21. C.A. Gandin, M. Rappaz, D. West and B.L. Adams, Grain Texture Evolution during the Columnar Growth of Dendritic Alloys, Metall. Mater. Trans. A, 1995, 26(6), p 1543-1551. https://doi.org/10.1007/ BF02647605

22. M. Rappaz and C.-A. Gandin, Probabilistic Modelling of Microstructure Formation in Solidification Processes, Acta Metall. Mater., 1993, 41(2), p 345-360. https://doi.org/10.1016/0956-7151(93)90065-Z
23. C.A. Gandin, M. Rappaz and R. Tintillier, 3-Dimensional Simulation of the Grain Formation in Investment Castings, Metall. Mater. Trans. A, 1994, 25(3), p 629-635.

24. M. Anderson, D.J. Srolovitz, G.S. Grest and P.S. Sahni, Computer Simulation of Grain Growth - I Kinetics, Acta Met., 1984, 5, p 783791.

25. D. Srolovitz, M. Anderson, P. Sahni and G. Grest, Computer Simulation of Grain Growth-II. Grain Size Distribution, Topology, and Local Dynamics, Acta Metall., 1984, 32(5), p 793-802. https://doi. org/10.1016/0001-6160(84)90152-4

26. D.J. Srolovitz, M.P. Anderson, G.S. Grest and P.S. Sahni, Computer Simulation of Grain Growth-III. Influence of a Particle Dispersion, Acta Metall., 1984, 32(9), p 1429-1438. https://doi.org/10.1016/00016160(84)90089-0

27. T.M. Rodgers, J.D. Madison, V. Tikare and M.C. Maguire, Predicting Mesoscale Microstructural Evolution in Electron Beam Welding, Jom, 2016, 68(5), p 1419-1426.

28. T.M. Rodgers, J.E. Bishop and J.D. Madison, Direct Numerical Simulation of Mechanical Response in Synthetic Additively Manufactured Microstructures, Model. Simul. Mater. Sci. Eng., 2018, 26(5), p 055010 .

29. D. Rosenthal, "Mathematical Theory of Heat Distribution During Wleding and Cutting," Welding Research Supliment, 1941, p 220-234

30. D.J. Rowenhorst, L. Nguyen, A.D. Murphy-Leonard and R.W. Fonda, Characterization of Microstructure in Additively Manufactured 316L Using Automated Serial Sectioning, Curr. Opin. Solid State Mater. Sci., 2020, 24(3), p 100819.

31. M.A. Groeber and M.A. Jackson, DREAM. 3D: A Digital Representation Environment for the Analysis of Microstructure in 3D, Integr. Mater. Manuf. Innov., 2014, 3(1), p 5.

32. L. Thijs, K. Kempen, J.-P. Kruth and J. Van Humbeeck, FineStructured Aluminium Products with Controllable Texture by Selective Laser Melting of Pre-Alloyed AlSi10Mg Powder, Acta Mater., 2013, 61(5), p 1809-1819. https://doi.org/10.1016/j.actamat.2012.11.052

33. X. Zhou, K. Li, D. Zhang, X. Liu, J. Ma, W. Liu and Z. Shen, Textures Formed in a CoCrMo Alloy by Selective Laser Melting, J. Alloys Compd., 2015, 631, p 153-164. https://doi.org/10.1016/j.jallcom.2015. 01.096

34. T. Ishimoto, K. Hagihara, K. Hisamoto, S.-H. Sun and T. Nakano, Crystallographic Texture Control of Beta-Type Ti-15Mo-5Zr-3Al Alloy by Selective Laser Melting for the Development of Novel Implants with a Biocompatible Low Young's Modulus, Scr. Mater., 2017, 132, p 34-38. https://doi.org/10.1016/j.scriptamat.2016.12.038

35. J.K. Mackenzie, Second Paper on Statistics Associated with the Random Disoreintation of Cubes, Biometrika, 1958, 45(1-2), p 229240. https://doi.org/10.1093/biomet/45.1-2.229

36. R.A. Lebensohn, A.K. Kanjarla and P. Eisenlohr, An Elasto-Viscoplastic Formulation Based on Fast Fourier Transforms for the Prediction of Micromechanical Fields in Polycrystalline Materials, Int. J. Plast., 2012, 32, p 59-69.

37. E. Voce, A Practical Strain-Hardening Function, Metallugia, 1955, 51, p 219-226.

38. Z. Wang, A.D. Stoica, D. Ma and A.M. Beese, Diffraction and SingleCrystal Elastic Constants of Inconel 625 at Room and Elevated Temperatures Determined by Neutron Diffraction, Mater. Sci. Eng. A, 2016, 674, p 406-412.

39. M.K. Hu, Visual Pattern Recognition by Moment Invariants, IRE Trans. Inf. Theory, 1962, 8(2), p 179-187.

40. J.P. MacSleyne, J.P. Simmons and M. De Graef, On the Use of 2-D Moment Invariants for the Automated Classification of Particle Shapes, Acta Mater., 2008, 56(3), p 427-437.

Publisher's Note Springer Nature remains neutral with regard to jurisdictional claims in published maps and institutional affiliations. 